\title{
HER-2/neu expression is associated with high tumor cell proliferation and aggressive phenotype in a population based patient series of endometrial carcinomas
}

\author{
INGEBORG B. ENGELSEN ${ }^{1,8}$, INGUNN M. STEFANSSON ${ }^{2}$, RAMEEN BEROUKHIM $^{3-5}$, WILLIAM R. SELLERS ${ }^{6}$, \\ MATTHEW MEYERSON ${ }^{3-5}$, LARS A. AKSLEN ${ }^{2,7}$ and HELGA B. SALVESEN ${ }^{1,8}$

\begin{abstract}
Departments of ${ }^{1}$ Obstetrics and Gynecology and ${ }^{2}$ Pathology, Haukeland University Hospital 2, 5021 Bergen, Norway;
${ }^{3}$ Department of Medical Oncology, Dana-Farber Cancer Institute, ${ }^{4}$ Broad Institute of Harvard and ${ }^{5}$ MIT, Boston, MA;

${ }^{6}$ Novartis Institutes of BioMedical Research, Cambridge, MA, USA; ${ }^{7}$ The Gade Institute, Section of Pathology,
\end{abstract} \\ ${ }^{8}$ Institute of Clinical Medicine, University of Bergen, 5020 Bergen, Norway
}

Received October 1, 2007; Accepted November 30, 2007

\begin{abstract}
Gene alterations and overexpression of various oncogenes and cell-cycle regulators are important in tumor development. In a population based series of 316 endometrial carcinomas with long and complete follow-up we investigated the distribution of HER-2/neu and EGFR expression and copy number alteration in endometrial cancers. HER-2/ neu, EGFR and Ki-67 expression in curettage and hysterectomy specimens were studied immunohistochemically for expression in relation to molecular markers and clinical phenotype. Fresh tumor samples $(n=76)$ were studied by global characterization of genetic alterations by single nucleotide polymorphism (SNP) array for detection of high level amplification for HER-2/neu and EGFR. Pathological expression of HER-2/neu in curettage was detected in $23 \%$ which significantly correlated to high FIGO stage, nonendometrioid subtype, high grade and aneuploidy. In hysterectomy specimens, pathological HER-2/neu staining was seen in $13 \%$ which correlated significantly with high FIGO stage, non-endometrioid subtype, high proliferation and poor survival $(\mathrm{p}=0.009)$. Expression of EGFR was examined with three different antibodies, but none showed significant correlation with molecular markers or clinical phenotype. High level amplification of HER-2/neu or EGFR was seen in only one out of 76 samples, respectively. High proliferation estimated in tumors from hysterectomy specimens showed independent prognostic impact and was superior to estimation in curettage specimens as a prognostic
\end{abstract}

Correspondence to: Dr Ingeborg Bøe Engelsen, Department of Obstetrics and Gynecology, Haukeland University Hospital, 5021 Bergen, Norway

E-mail: iben@helse-bergen.no

Key words: endometrial carcinoma, curettage, tumor markers, HER-2/neu, proliferation marker. In conclusion, high level amplification of HER-2/neu or EGFR is infrequent in endometrial cancer. Pathological HER-2/neu staining identifies endometrial carcinomas with an aggressive phenotype, high proliferation and patients with poor survival in a population based setting. These results motivate further clinical trials with trastuzumab based on HER-2/neu status in endometrial carcinomas.

\section{Introduction}

Endometrial carcinoma is presently the most common gynecologic malignancy in industrialized countries and the incidence is increasing. Risk factors predisposing for endometrial cancer are germline mutations in DNA repair genes, Hereditary Non-Polyposis Colorectal Cancer syndrome (HNPCC), nulliparity, obesity, diabetes mellitus and tamoxifen use (1). Despite high curability of endometrial cancer, tumors with adverse histopathological features and advanced stage are characterized by aggressive behaviour and poor prognosis (1).

Over the years, several reports on endometrial cancer and prognostic factors have been published and the impact of age, histologic type and grade, FIGO stage, ploidy and hormone receptor status is well established (1). The molecular pathogenesis of endometrial carcinoma remains poorly understood, but studies have shown that malignant tumors arise by alterations in genes controlling proliferation, apoptosis, angiogenesis, invasion, and metastases (2). A better understanding of these events may facilitate improved diagnosis and development of new drugs aimed at specific molecular targets (3).

Endometrial tumors are easily accessible for evaluation prior to primary surgery through curettage material and some of these prognostic markers can potentially be used to facilitate early diagnosis. Thus, improved markers for prognostication may make extensive and potentially harmful surgical staging unnecessary in low-risk patients and improve staging of the disease and planning of individualized treatment among high-risk patients (4). 
New and effective treatments for patients with metastatic endometrial carcinoma are needed while there is no therapy with documented effect on survival in randomized controlled trials (3). Treatment with targeted therapies shows promising results in cancer types such as breast (trastuzumab), colon (cetuximab) and lung (erlotinob) $(5,6)$. The proto-oncogenes, HER-2/neu and EGFR, both members of the epidermal growth factor receptor tyrosine kinase family, have an important role in regulating cell growth and differentiation, although it is not clear how a specific signalling pathway corresponds to biological and clinical response (7). HER-2/ neu overexpression has been associated with a metastatic phenotype and poor survival in different cancer types (8). Trastuzumab (Herceptin, Roche, Basel, Switzerland) is a monoclonal antibody that binds to HER-2/neu receptors and suppresses tumor growth. Santin et al, showed that HER-2/neu was highly expressed in uterine serous papillary carcinoma (USPC) and that the USPC cells were sensitive to trastuzumab therapy (9). The effect of EGFR inhibition in endometrial cancer cell lines seems to be more limited (10).

On this background, we examined the occurrence of genetic alteration as well as the expression patterns and prognostic significance of HER-2/neu and EGFR in a population based patient series with extensive follow-up data. In particular, we investigated the relationship to proliferative activity in the tumors and the prognostic value of these markers in curettage specimens for potential use as preoperative indicators for an aggressive phenotype.

\section{Materials and methods}

All 316 patients with endometrial carcinoma in Hordaland County, Norway, occurring from 1981 to 1990 were studied retrospectively (11). Several clinico-pathological variables were recorded including International Federation of Gynecology and Obstetrics (FIGO) stage (1), histologic subtype and grade, treatment and disease specific survival (11). A panel of tumor markers investigated in the hysterectomy specimens was available for comparison; such as the presence of necrosis, mitotic count, vascular invasion, DNAindex, estrogen receptor (ER), progesterone receptor (PR), p53 and p16 expression. Data have been presented elsewhere previously (11-13).

Of the 316 patients diagnosed with endometrial carcinoma in the study period, 12 were excluded due to changed diagnosis at reclassification and five due to a diagnosis based on cytological examination only (12). Of the remaining 299 cases, paraffin blocks from primary tumor were available in 286 cases $(96 \%)$ and from curettage specimens in 236 cases (79\%). The number of cases with evaluable results varied for the different markers studied. In order to preserve statistical power we have chosen to present all the data available for each marker. The research was approved by the Norwegian Data Inspectorate (961478-2) and the Local Ethics Committee (REK III nr.052.01).

Tissue micro-array (TMA). The TMA technique has previously been described and validated in several studies. Briefly, TMA construction was made by identifying the area of highest tumor grade on hematoxylin and eosin stained slides, followed by punching out three tissue cylinders with a diameter of $0.6 \mathrm{~mm}$ from the selected areas of the donor block and mounted into a recipient paraffin block using a custom-made precision instrument (Beecher Instruments, Silver Spring, MD). Sections of the resulting TMA blocks $(5-\mu \mathrm{m})$ were made by standard technique. A parallel series of TMA blocks were made from tumor tissue from curettageand hysterectomy specimens in the series. Curettage $(n=200)$ and hysterectomy $(n=230)$ samples had representative tissue in the TMA sections used for analyses. Ki-67 and HER-2/neu expression were investigated in curettage and hysterectomy specimens, while EGFR expression was studied in curettage material only.

We investigated the potential selection bias in the patient series with representative tumor tissue in TMA blocks from curettage or hysterectomy specimens. The distribution of FIGO stage and survival among these patients was similar to the rest of the patients from Hordaland County treated for endometrial carcinoma during the same period from whom representative curettage or hysterectomy specimens were not available in the TMA blocks ( $n=99$ and $n=69$ respectively).

SNP array. A separate population based series of 76 endometrial cancers prospectively collected was used for global characterization of genetic alterations (amplifications and deletions) by SNP (single nucleotide polymorphism) array. This method detects copy number alterations and loss of heterozygosity (14). Samples with high level amplification were stained immunohistochemically and served as positive controls for membranous staining for HER-2/neu and EGFR overexpression.

Immunohistochemistry. For Ki-67 and HER-2/neu microwave epitope retrieval was used $(10 \mathrm{~min}, 750 \mathrm{~W} ; 15 \mathrm{~min}, 350 \mathrm{~W}$ in Tris-(hydroxymethyl) aminomethane-EDTA buffer; $\mathrm{pH} 9.0$ ). EGFR had pretreatment with protein kinase (10 min). The staining was performed on the Dako Cytomatation Autostainer for Ki-67 and HER-2/neu and manually for EGFR. All slides were blocked with peroxidase (S-2023) for $5 \mathrm{~min}$ followed by incubation with the different antibodies at room temperature for $1 \mathrm{~h}$ when not otherwise specified. We used monoclonal mouse Ki-67 antibody (M 7240, Dako, Copenhagen, Denmark), diluted 1:250 with 30-min incubation and polyclonal rabbit HER-2/neu antibody (A-0485, Dako), diluted 1:500. For EGFR we used three different antibodies, the monoclonal mouse EGFR antibody (A-431, clone 31G7, Zymed), diluted 1:30 with 30-min incubation, the polyclonal rabbit antibody (A-3105, sc-03, Santa Cruz), diluted 1:400 and finally the EGFR pharm DX kit (K1494-Dako) in the Dako autostainer. The antibodies were localized by the EnVision chain-polymer method (K 5007, DakoCytomation, Copenhagen, Denmark) with diaminobezidine peroxidase (DAB) as a substrate prior to counterstaining with Harris hematoxylin.

Evaluation of staining. Blinded to patient characteristics and outcome, the slides were evaluated for immunohistochemical staining and scored by two of the authors (I.B. Engelsen and H.B. Salvesen). Membranous staining for HER-2/neu was scored according to the Dako Hercep test criteria (15). The 
membrane staining intensity and pattern was recorded for HER-2/neu in line with previous studies of breast cancer giving a score from 0 to 3 ; no staining or membrane staining $<10 \%$ (0), faint partial staining of the membrane in $>10 \%$ (1), weak (2) or strong (3) staining of the entire membrane in $>10 \%$ of the tumor cells. For evaluation of EGFR membranous and cytoplasmic staining (Zymed and Dako) we used a staining-index (SI) including both staining intensity and area for two different antibodies (Zymed and Dako). Staining intensity was graded from 0 (no staining) to 3 (strong staining). The percentage of staining area was graded as 0 (no staining), 1 (staining in $<10 \%$ ), 2 (staining in 10 $50 \%$ ) or 3 (staining in $>50 \%$ ). The SI was calculated as the product of staining intensity and staining area. For the third EGFR antibody explored (Santa Cruz) we registered nuclear staining using SI. No membranous staining was seen for this antibody. Two observers recorded HER-2/neu and EGFR staining (I.B. Engelsen and H.B. Salvesen).

Ki-67 staining was assessed according to the 'hot-spot' approach of Weidner et al as previously reported (12). The most intense staining area was identified and the percentage of positive nuclei was calculated by counting 500 tumor cells at magnification $\mathrm{x} 1000$. The Ki-67 estimations were done by one author (I.B. Engelsen) after a pilot study validating the reproducibility of the method (interobserver kappa coefficient 0.72 ).

Statistical analyses. Analyses were performed by SPSS 11.0. Associations were assessed by Pearson's $\chi^{2}$, Mann-Whitney and Kruskall-Wallis tests. Univariate analyses of time to death due to endometrial carcinoma were performed using the Kaplan-Meier method. Differences in survival were estimated by the log-rank (Mantel-Cox) test. The variables with significant impact on survival in univariate analyses $(\mathrm{p} \leq 0.05)$ were further examined by log-minus-log plot to decide how these variables could be incorporated in the Cox proportional hazards regression model. Unadjusted and adjusted hazard ratios were estimated as a measure of effect. In the statistical analyses, cut-off values for categorisation were based on median and quartiles, also considering the frequency distribution for each marker, the size of subgroups and number of events in each category. Groups with similar survival were merged.

For HER-2/neu expression the data were divided into two groups, negative staining (score 0-1) and positive staining (score 2-3) according to the Hercep test criterias. For EGFR expression (Zymed, Dako and Santa Cruz) we saw no difference in survival according to staining index and staining intensity analyzed separately. We decided to present the data from the Dako antibody using Dako criteria as they have been used in other studies (16). Briefly, Dako criteria divide the data into two groups, positive (score $>0$ ) and negative (score 0$)$.

For Ki-67 counts in curettage material, the two lower quartiles showed similar survival compared to the upper quartiles, and the median value was used to categorize into two groups, low $(<34 \%)$ versus high proliferation $(\geq 34 \%)$, (Table III and Fig. 3A).

For the hysterectomy specimens (12), the upper quartile $(\geq 35 \%)$ was used as cutpoint, in line with previous studies and with similar survival for the groups merged (Table III and Fig. 3B).

\section{Results}

HER-2/neu expression. Positive membranous staining for HER-2/neu in curettage material $(45 / 198 ; 23 \%)$ was significantly associated with high FIGO stage, serous papillary and clear cell tumors, high histological grade, presence of vascular invasion and aneuploidy as listed in Table I. Among the 185 endometrioid tumors, positive HER-2/neu expression was seen in 36 (20\%). Positive HER-2/neu staining in this histological subgroup was associated with high FIGO stage $(\mathrm{p}=0.03)$, aneuploidy $(\mathrm{p}=0.05)$ and loss of PR expression ( $\mathrm{p}=0.06$, borderline significance).

For HER-2/neu in 217 hysterectomy specimens we found positive HER-2/neu membranous expression in 28 cases (13\%). Membranous staining was significantly associated with high FIGO stage $(\mathrm{p}=0.05)$ and serous papillary and clear cell histological subtype $(\mathrm{p}<0.0001)$ as listed in Table I. Among the endometrioid tumors, positive membranous expression was seen in $10 \%(19 / 195)$, with no significant associations to any of the listed variables.

One of $76(1.3 \%)$ samples characterized for genetic alterations by SNP array showed high level amplification for HER-2/neu (case 185T; Fig. 1). This sample showed positive membranous staining for HER-2/neu and was used as a positive control for the immunohistochemical studies.

EGRF expression. The EGFR expression pattern was analyzed in curettage material only using three different EGFR antibodies and the results were correlated to clinico-pathological variables, different tumor markers and survival. For all three antibodies, we did not find any significant correlations, when examining the membrane pattern only (Dako) or when examining the cytoplasmic (Dako and Zymed) or nuclear (Santa Cruz) expression patterns separately (data not shown). The Dako and Zymed antibodies showed positive correlation for membranous staining $(\mathrm{r}=0.47 ; \mathrm{p}=0.0001)$ and cytoplasmic staining $(\mathrm{r}=0.21 ; \mathrm{p}=0.003)$. One of $76(1.3 \%)$ samples characterized for genetic alterations by SNP array showed high level amplification for EGFR (case 51T; Fig. 1). We observed strong membranous staining for EGFR in the case with high level EGFR amplification detected by SNP array (case 51T, Fig. 1). This sample showed positive membranous staining for EGFR for all antibodies as well as nuclear staining using the Santa Cruz antibody and it was used as positive control for further immunohistochemical studies. As positive EGFR expression for three different antibodies did not give any significant associations to clinico-pathological variables or biomarkers in curettage material, we decided not to further examine hysterectomy specimens for EGFR expression.

Tumor cell proliferation (Ki-67). The median nuclear expression of Ki-67 in curettage material was $34 \%(n=190)$ within the hot-spot areas. Ki-67 expression was significantly higher among tumors with high mitotic count, presence of necrosis and vascular invasion as listed in Table II. There were no significant associations with histological subtype, histological grade, FIGO stage or ploidy. In comparison, the 
Table I. HER-2/neu expression in preoperative curettage and hysterectomy specimens related to clinico-pathological variables and selected biomarkers in a population-based study of endometrial carcinoma patients.

\begin{tabular}{|c|c|c|c|c|c|c|c|}
\hline \multirow[b]{2}{*}{ Variables } & \multirow[b]{2}{*}{ Categories } & \multicolumn{3}{|c|}{$\begin{array}{l}\text { Expression in curettage specimens } \\
\qquad(\mathrm{n}=198)^{\mathrm{a}}\end{array}$} & \multicolumn{3}{|c|}{$\begin{array}{l}\text { Expression in hysterectomy specimens } \\
\qquad(\mathrm{n}=217)^{\mathrm{b}}\end{array}$} \\
\hline & & $\begin{array}{c}\text { Negative (0-1) } \\
\mathrm{n}(\%)\end{array}$ & $\begin{array}{l}\text { Positive (2-3) } \\
\text { n (\%) }\end{array}$ & p-value ${ }^{c}$ & $\begin{array}{c}\text { Negative }(0-1) \\
\mathrm{n}(\%)\end{array}$ & $\begin{array}{l}\text { Positive (2-3) } \\
\text { n (\%) }\end{array}$ & $\overline{p-\text { value }^{c}}$ \\
\hline \multirow[t]{2}{*}{ FIGO stage } & $\mathrm{I} / \mathrm{II}$ & $130(80)$ & $32(20)$ & 0.03 & $162(89)$ & $20(11)$ & 0.05 \\
\hline & III/IV & $22(63)$ & $13(37)$ & & 27 (77) & $8(23)$ & \\
\hline \multirow{2}{*}{$\begin{array}{l}\text { Histological } \\
\text { subtype }\end{array}$} & Endometroid & $149(81)$ & $36(19)$ & 0.0001 & $176(90)$ & $19(10)$ & $<0.0001$ \\
\hline & $\begin{array}{l}\text { Serous papillary/ } \\
\text { clear cell }\end{array}$ & $4(31)$ & 9 (69) & & $13(59)$ & $9(41)$ & \\
\hline \multirow{3}{*}{$\begin{array}{l}\text { Histological grade } \\
\text { (FIGO) }\end{array}$} & I & $39(89)$ & $5(11)$ & 0.007 & $46(90)$ & $5(10)$ & 0.18 \\
\hline & II & $92(79)$ & $25(21)$ & & $110(89)$ & $14(11)$ & \\
\hline & III & $22(60)$ & $15(40)$ & & $33(79)$ & $9(21)$ & \\
\hline \multirow[t]{2}{*}{ Necrosis } & Not present & $72(83)$ & $15(17)$ & 0.10 & $76(84)$ & $14(16)$ & 0.33 \\
\hline & Present & $81(73)$ & $30(27)$ & & $113(89)$ & $14(11)$ & \\
\hline \multirow[t]{2}{*}{ Mitotic count } & $<$ Median $^{\mathrm{d}}$ & $86(78)$ & $24(22)$ & 0.70 & $115889)$ & $15(11)$ & 0.44 \\
\hline & $\geq$ Median & $66(76)$ & $21(24)$ & & $73(85)$ & $13(15)$ & \\
\hline Vascular & 0 or 1 vessel & $101(82)$ & $22(18)$ & 0.04 & $114(88)$ & $16(12)$ & 0.75 \\
\hline invasion & $>1$ vessels & $52(69)$ & $23(31)$ & & $75(86)$ & $12(14)$ & \\
\hline \multirow[t]{2}{*}{ DNA ploidy } & Diploid & $50(89)$ & $6(11)$ & 0.02 & $55(90)$ & $6(10)$ & 0.14 \\
\hline & Aneuploid & $9(64)$ & $5(36)$ & & $13(77)$ & $4(23)$ & \\
\hline \multirow[t]{2}{*}{ p53 } & Normale & $78(80)$ & $19(20)$ & 0.15 & $109(91)$ & 11 (9) & 0.85 \\
\hline & Pathologic & 17 (94) & 1 (6) & & $26(90)$ & $3(10)$ & \\
\hline \multirow[t]{2}{*}{ p16 } & Normalf $^{\mathrm{f}}$ & $115(78)$ & $32(22)$ & 0.65 & $163(87)$ & $24(13)$ & 0.94 \\
\hline & Pathologic & $14(74)$ & $5(26)$ & & $26(87)$ & $4(13)$ & \\
\hline \multirow[t]{2}{*}{ ER expression } & Normal & $102(80)$ & $25(20)$ & 0.30 & $140(88)$ & $19(12)$ & 0.40 \\
\hline & Pathologic & $26(72)$ & $10(28)$ & & $46(84)$ & $9(16)$ & \\
\hline \multirow[t]{2}{*}{ PR expression } & Normal & $96(82)$ & $21(18)$ & 0.13 & $133(89)$ & $17(11)$ & 0.39 \\
\hline & Pathologic & $32(71)$ & $13(29)$ & & $54(84)$ & $10(16)$ & \\
\hline
\end{tabular}

${ }^{a}$ Missing data in 1 case for FIGO stage and mitotic count, 128 cases for DNA ploidy, 83 cases for p53, 32 cases for p16, 35 cases for ERand 36 cases for PR expression. ${ }^{b}$ Missing data in 1 case for mitotic count, 139 cases for DNA ploidy, 68 cases for p53, - , 3 cases for p16and 3 cases for ER expression. ${ }^{c} \chi^{2}$ test. ${ }^{d}$ Low mitotic count is less than the median value and high mitotic count is more or equal to the median. ${ }^{e}$ Normal p53 expression index was $\leq 4$ and pathologic p53 expression index was $>4$. ${ }^{\mathrm{f}}$ Normal p16 expression index was $\geq 4$ and pathologic p16 expression index was $<4$.

median expression for Ki-67 in hysterectomy specimens for the same patients was $17 \%(n=230)$. High Ki-67 expression in hysterectomy specimens showed a significant correlation with high FIGO stage, serous papillary/clear cell histological subtype, poor histological grade and high mitotic count (Table II). The expression of Ki-67 in curettage material was highly significantly correlated to Ki-67 expression in the corresponding hysterectomy specimens (Pearson's correlation coefficient, 0.39; $\mathrm{p}=0.007)$. The estimated linear regression line and observed values are shown in Fig. 2.

We recorded fixation time in days for 99 cases of curettage and hysterectomy specimens and found a median of 3 and 


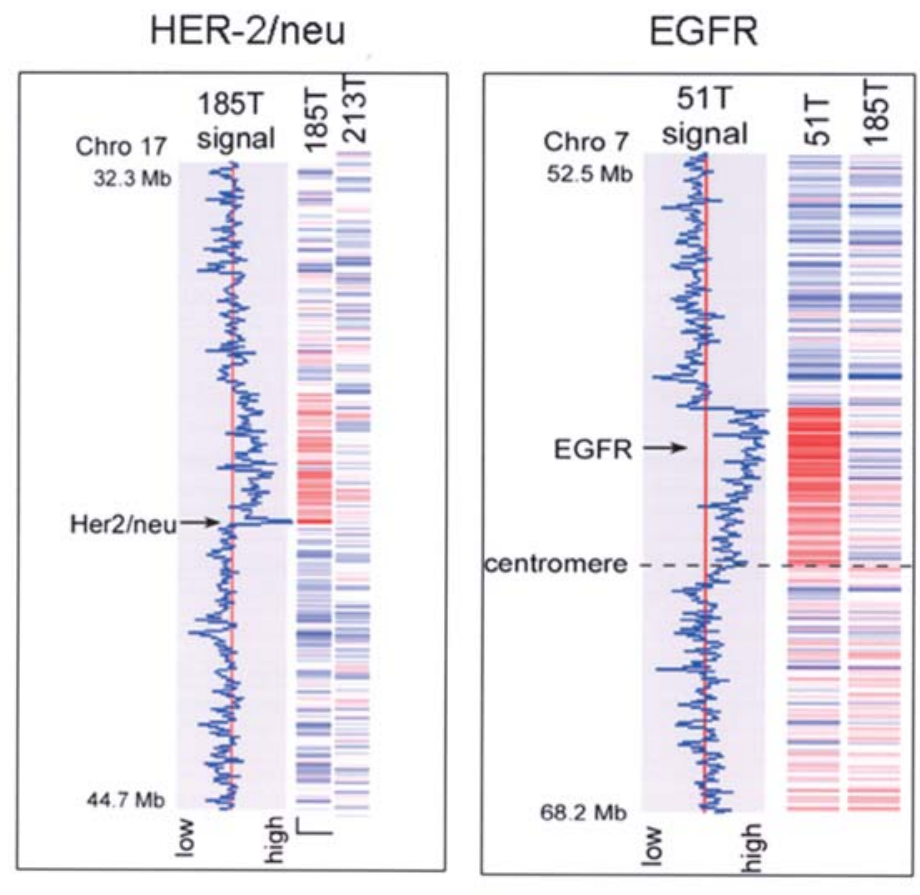

SNP-ARRAY
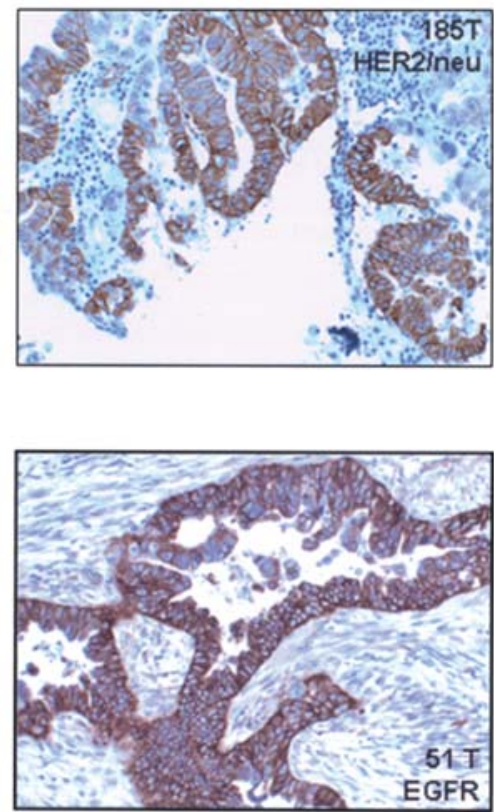

IMMUNOHISTOCHEMISTRY

Figure 1. High level amplification of HER-2/neu by SNP array was seen in 1 of 76 investigated samples (185T). Immunohistochemical staining for HER-2/neu confirmed positive membranous staining for the same tumor. High level amplification of EGFR by SNP array was seen in 1 of 76 samples (51T). Immunohistochemical staining for EGFR (Dako-Ab) confirmed positive membranous staining for the same tumor sample.

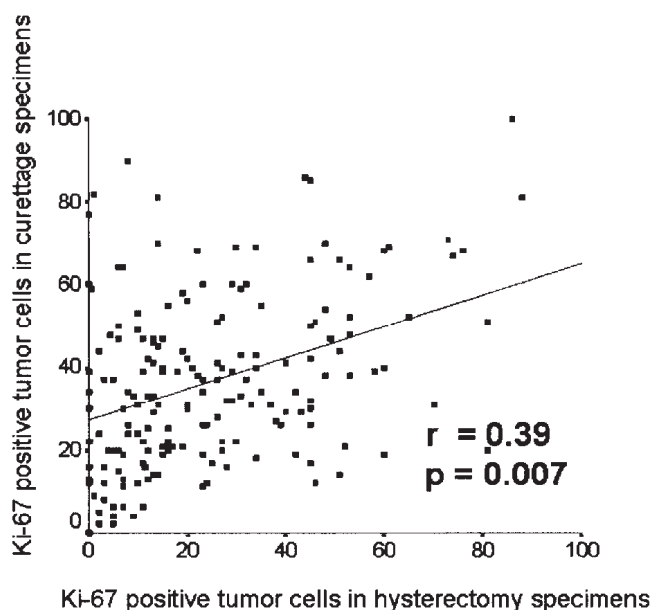

Figure 2. Scatter plot of tumor cell proliferation by Ki-67 staining in curettage material and $\mathrm{Ki}-67$ staining in hysterectomy specimens. A regression line is drawn to illustrate the relationship.

4 days respectively, although no significant difference in Ki-67 expression according to fixation time.

Associations with other biomarkers. Data on selected biomarkers were included from previous studies for comparisons with our staining data for HER-2/neu, EGFR and Ki-67 expression $(11,13,17)$. Briefly, positive HER-2/neu expression in curettage showed a significant association with low expression of $\beta$-catenin $(\mathrm{p}=0.002)$, high expression of $\mathrm{P}$ cadherin $(p=0.001)$, presence of glomeruloid microvascular proliferation $(\mathrm{p}=0.05)$ and vessel infiltration $(\mathrm{p}=0.04)$. Positive
HER-2/neu staining in hysterectomy specimens was associated with high membranous expression of P-cadherin $(\mathrm{p}=0.01)$ and high Ki-67 expression in tumors (0.04).

High Ki-67 expression in curettage was associated to loss of estrogen receptors $(\mathrm{ER})(\mathrm{p}=0.002)$ and progesterone receptors $(P R)(p<0.0001)$ as well as pathologic p53 $(\mathrm{p}=0.05)$ and $\mathrm{p} 16(\mathrm{p}<0.0001)$ expression, whilst high Ki-67 expression in tumors from hysterectomy specimens was associated with loss of PR staining $(\mathrm{p}=0.005)$. For EGFR, there was no association to any of the markers

Survival analysis. In the univariate analysis, Ki-67 expression in both curettage and hysterectomy specimens was significantly associated with patient survival (Table III and Fig. 3A and B). Positive staining for HER-2/neu in tumors from hysterectomy specimens was significantly associated with poor survival ( $p=0.009$; Fig. 3D), while HER-2/neu staining in curettage specimens did not influence survival significantly $(\mathrm{p}=0.18$, Fig. 3C). The EGFR expression did not influence survival significantly for any of the antibodies used on curettage samples.

The prognostic impact of HER-2/neu and Ki-67 expression was tested in the multivariate Cox regression analysis including patient age at diagnosis, FIGO stage, histologic subtype and histologic grade. Ki-67 expression in curettage specimens showed no independent prognostic influence. Ki-67 expression estimated in primary tumors from hysterectomy specimens when available, had independent prognostic impact in a forward stepwise regression analysis with an HR of 1.9 (95\% CI 1.1-3.1, p=0.02). For HER-2/neu however, there was no independent prognostic impact. 
Table II. Ki-67 expression in preoperative curettage and subsequent hysterectomy specimens related to clinico-pathological variables and selected biomarkers in a population-based study of endometrial carcinoma patients.

\begin{tabular}{|c|c|c|c|c|c|}
\hline \multirow[b]{2}{*}{ Variables } & \multirow[b]{2}{*}{ Categories } & \multicolumn{2}{|c|}{$\begin{array}{c}\text { Nuclear Ki-67 expression } \\
\text { in curettage } \\
\text { specimens }(\mathrm{n}=190)^{\mathrm{a}}\end{array}$} & \multicolumn{2}{|c|}{$\begin{array}{c}\text { Nuclear Ki-67 expression } \\
\text { in hysterectomy } \\
\text { specimens }(n=230)^{b}\end{array}$} \\
\hline & & Median (n) & $\mathrm{p}$-value ${ }^{\mathrm{c}}$ & Median (n) & $\mathrm{p}$-value $\mathrm{c}^{\mathrm{c}}$ \\
\hline \multirow[t]{2}{*}{ FIGO stage } & $\mathrm{I} / \mathrm{II}$ & $33(158)$ & 0.13 & $15(193)$ & 0.02 \\
\hline & III/IV & $41 \quad(31)$ & & $31 \quad(37)$ & \\
\hline \multirow[t]{2}{*}{ Histologic subtype } & Endometrioid & 34 (176) & 0.40 & $16(210)$ & 0.01 \\
\hline & Serous papillary/clear cell & 36 (14) & & $34(20)$ & \\
\hline \multirow[t]{3}{*}{ Histologic grade (FIGO) } & I & 31 (44) & $0.12^{\mathrm{d}}$ & $15 \quad(53)$ & $0.002^{\mathrm{d}}$ \\
\hline & II & 34 (109) & & $15(133)$ & \\
\hline & III & 39 (37) & & 27 (44) & \\
\hline \multirow[t]{2}{*}{ Necrosis } & Not present & $30 \quad(82)$ & 0.004 & $13(91)$ & 0.10 \\
\hline & Present & 39 (108) & & 19 (139) & \\
\hline \multirow[t]{2}{*}{ Mitotic count } & Low $^{\mathrm{e}}$ & $27(105)$ & 0.0001 & $13(139)$ & 0.0001 \\
\hline & High & $39(84)$ & & $29(90)$ & \\
\hline \multirow[t]{2}{*}{ Vascular invasion } & 0 or 1 vessel & $31(118)$ & 0.02 & $17(136)$ & 0.40 \\
\hline & $>1$ vessels & $40 \quad(72)$ & & $18 \quad(94)$ & \\
\hline \multirow[t]{2}{*}{ DNA ploidy } & Diploid & $31 \quad(50)$ & 0.38 & $15 \quad(65)$ & 0.21 \\
\hline & Aneuploid & 32 (14) & & $30 \quad(17)$ & \\
\hline \multirow[t]{2}{*}{ p53 expression } & Normal $^{\mathrm{f}}$ & 33 (113) & 0.05 & $16(126)$ & 0.06 \\
\hline & Pathologic & $43 \quad(27)$ & & $31 \quad(33)$ & \\
\hline \multirow[t]{2}{*}{ p16 expression } & Normal' & 31 (167) & $<0.0001$ & 17 (200) & 0.19 \\
\hline & Pathologic & $50 \quad(23)$ & & $28 \quad(30)$ & \\
\hline \multirow[t]{2}{*}{ ER expression } & Normal & 33 (122) & 0.002 & $16(168)$ & 0.12 \\
\hline & Pathologic & 43 (34) & & $25 \quad(58)$ & \\
\hline \multirow[t]{2}{*}{ PR expression } & Normal & 30 (113) & $<0.0001$ & $15(158)$ & 0.01 \\
\hline & Pathologic & $47 \quad(42)$ & & $30 \quad(68)$ & \\
\hline \multirow[t]{2}{*}{ HER-2/neu } & Negative $(0-1)$ & $13(160)$ & 0.013 & $16(185)$ & 0.03 \\
\hline & Positive (2-3) & 33 (20) & & $35 \quad(27)$ & \\
\hline
\end{tabular}

${ }^{a}$ Missing data in 1 case for FIGO stage and mitotic count, 2 cases for p16, 34 cases for ER, 35 cases for PR and 50 cases for p53 expression, 10 cases for HER-2/neu and 126 cases for DNA ploidy. ${ }^{b}$ Missing data in 1 case for mitotic count, 71 cases for p53, 4 cases for ER and PR expression, 18 cases for HER-2/neu and 148 cases in DNA ploidy. ${ }^{c}$ Mann-Whitney test. ${ }^{\mathrm{d}}$ Kruskall-Wallis test. ${ }^{\mathrm{e}}$ Low mitotic count is less than the median value and high mitotic count is more or equal to the median. ${ }^{\mathrm{f}}$ Normal expression index was $\leq 4$ and pathologic expression index was $>4$ (1). "Pathologic expression index was $<4$ and normal expression index was $\geq 4$ (1).

\section{Discussion}

Gene alterations and overexpression of various oncogenes and cell-cycle regulators have in earlier reports shown prognostic importance in endometrial cancer $(11,12,18,19)$.
High-level amplification of the HER-2/neu gene seems to be infrequent in endometrial carcinomas. In the prospective series of 76 cases examined for amplification by SNP array, only one case $(1.3 \%)$ was found to show a clear high-level amplification of HER-2/neu. Even though this sample showed 
Table III. Cause specific survival for 286 patients with endometrial carcinoma (Kaplan-Meier method) according to clinicopathologic variables and expression of Ki-67, HER-2/neu and EGFR. ${ }^{a}$

\begin{tabular}{|c|c|c|c|c|}
\hline Variables & $\mathrm{n}$ & $\begin{array}{l}\text { No. of } \\
\text { deaths }\end{array}$ & $\begin{array}{l}\text { Estimated 5-year } \\
\quad \text { survival (\%) }\end{array}$ & $\mathrm{p}$-value ${ }^{\mathrm{b}}$ \\
\hline FIGO stage & & & & $<0.0001$ \\
\hline I & 211 & 24 & 90 & \\
\hline II & 19 & 8 & 62 & \\
\hline III & 42 & 24 & 37 & \\
\hline IV & 13 & 13 & 0 & \\
\hline Histological subtype & & & & $<0.0001$ \\
\hline Endometrioid $^{c}$ & 257 & 53 & 80 & \\
\hline Clear cell/serous papillary & 29 & 17 & 38 & \\
\hline Histological grade (FIGO) & & & & $<0.0001$ \\
\hline Grade 1 & 64 & 5 & 92 & \\
\hline Grade 2 & 163 & 41 & 76 & \\
\hline Grade 3 & 59 & 24 & 59 & \\
\hline HER-2/neu expression in curettage & & & & 0.18 \\
\hline Negative (0-1) & 153 & 33 & 80 & \\
\hline Positive (2-3) & 45 & 13 & 70 & \\
\hline HER-2/neu expression in hysterectomy specimens & & & & 0.009 \\
\hline Negative (0-1) & 189 & 38 & 81 & \\
\hline Positive (2-3) & 28 & 11 & 61 & \\
\hline Ki-67 expression in curettage & & & & 0.03 \\
\hline Low & 93 & 15 & 84 & \\
\hline High & 97 & 30 & 71 & \\
\hline Ki-67 expression in hysterectomy specimens & & & & 0.01 \\
\hline Low & 176 & 33 & 82 & \\
\hline High & 54 & 18 & 66 & \\
\hline EGFR expression in curettage & & & & 0.67 \\
\hline Negative (0) & 135 & 32 & 77 & \\
\hline Positive (>1) & 65 & 14 & 79 & \\
\hline
\end{tabular}

${ }^{a}$ Missing data for 1 case in FIGO stage, 88 cases for HER-2/neu in curettage, 69 cases for HER-2/neu in hysterectomy specimens, 96 cases for Ki-67 expression in curettage, 56 cases for Ki-67 expression in hysterectomy specimens and 86 cases for EGFR expression in curettage. ${ }^{b}$ Log-rank (Mantel Cox) test for difference in survival between groups. ${ }^{c}$ Adenosquamous/adenoacantomas are included.

distinct immunohistochemical overexpression of HER-2/neu, an alteration in gene copy numbers estimated by SNP array does not seem to explain the mechanism for immunohistochemical overexpression observed in a much larger proportion of tumors in the independent and larger series studied by immunohistochemistry. This seems to be in line with previous reports showing discordance between amplification and immunohistochemical expression $(22,24)$, indicating that other mechanisms may be involved. The reported prevalence of HER-2/neu overexpression by immunohistochemistry varies from 9 to $30 \%$ in previous endometrial carcinoma studies. Overexpression has been reported to be more frequent among non-endometrioid tumors (18-80\% positivity) and has also been linked to poor survival (20). The variable prevalence of HER-2/neu expression in the literature could be explained by differences in patient populations, criteria for case selection, technical methods, other antibodies and in the interpretation of staining results.

Our study is the first to address this in population based patient series. We found that $23 \%$ of these tumors over- 

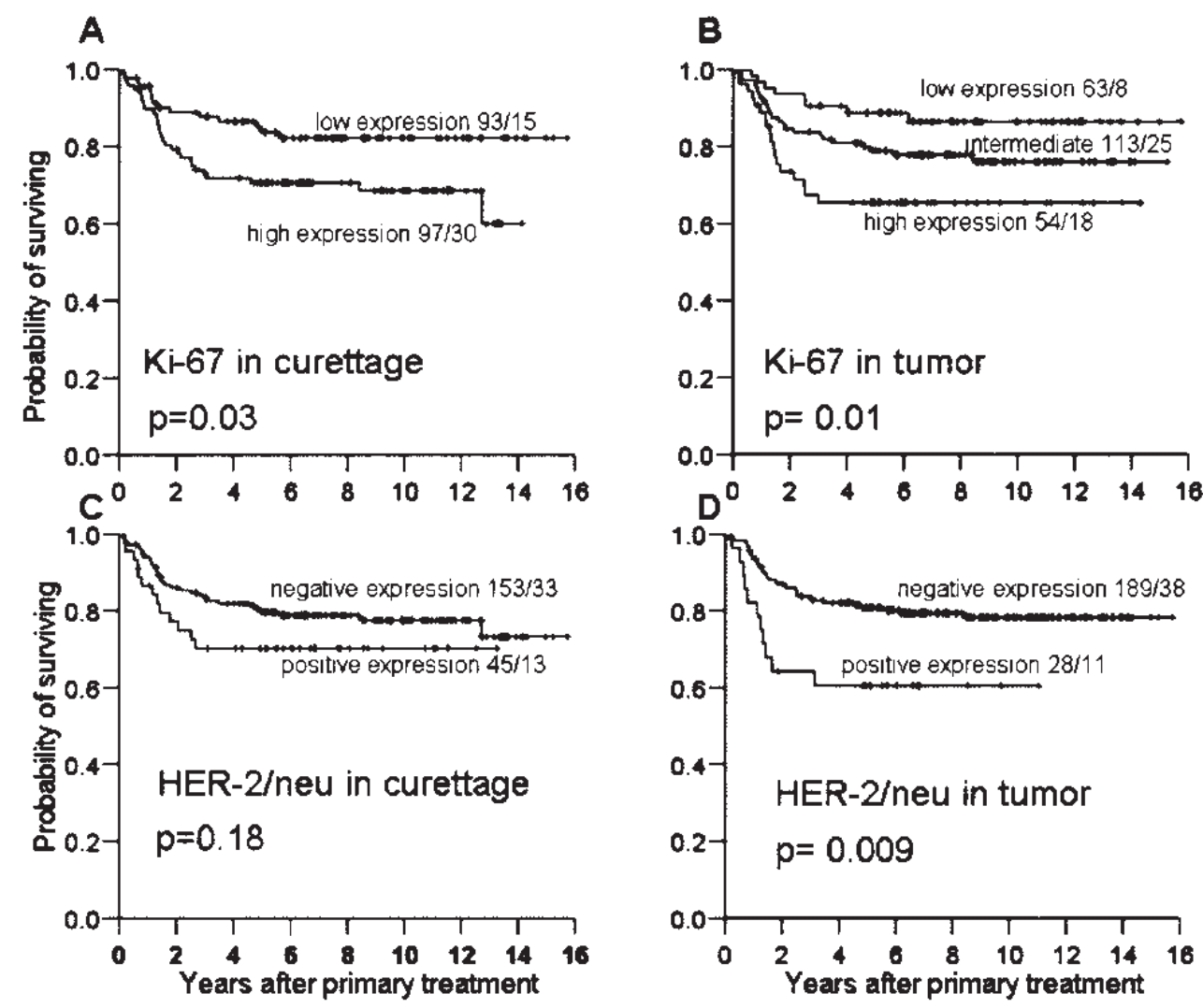

Figure 3. Estimated survival among patients with endometrial carcinoma according to (A) Ki-67 expression (Kaplan-Meier method) in tumor from curettage specimens, (B) Ki-67 expression in tumor from hysterectomy specimens, (C) HER-2/neu expression in curettage specimens, (D) HER-2/neu expression in tumor from hysterectomy specimens. For each category the number of cases is given followed by the number of cancer deaths.

expressed HER-2/neu in curettage material which appears to be in the upper range of what has been reported previously (21). In a large hospital based study, Morrison et al recently found a $14 \%$ prevalence of positive immunohistochemical HER-2/neu expression in tumor specimens (22) and is more in line with our $13 \%$ overexpression in hysterectomy specimens.

For curettage and hysterectomy material we found a strong association with several clinico-pathological and molecular markers such as FIGO stage and histological subtype, and for curettage, also with histological grade and DNA ploidy. This confirms that HER-2/neu may be an important tumor biological factor in a substantial proportion of endometrial carcinomas. Our finding that $69 \%$ of the serous papillary/clear cell tumors showed overexpression of HER-2/neu in curettage specimens indicate that this marker can be used to facilitate individualized treatment with preoperative referral to specialized cancer units for more extensive treatment of aggressive histologic subtypes. Endometrial carcinoma is known to be a hormone-responsive neoplasm. An inverse correlation between HER-2/ neu expression and the receptors for estrogen and progesterone was not seen in our study as has been shown in previous reports (21).

In line with other reports, HER-2/neu overexpression in hysterectomy specimens showed prognostic impact (22), but in contrast to the report of Mariani et al, the prognostic impact of HER-2/neu overexpression in curettage material did not reach statistical significance in our patient series.
This difference can be explained by the number of cases and the differences in populations studied $(22,23)$. The lack of independent prognostic impact of HER-2/neu overexpression previously reported, appear to be in line with our study though (23).

Cell line studies of endometrial carcinoma have shown that efficacy of trastuzumab therapy is correlated to HER-2/neu overexpression in serous papillary cancer (9). This could represent a novel and effective therapy for metastatic endometrial carcinomas overexpressing HER-2/neu (9), and a phase II evaluation of trastuzumab in recurrent and advanced endometrial carcinomas is now underway (GOG trial 181B). In the future, a molecularly based therapy regimen might be used for treatment of HER-2/neu overexpressing metastatic endometrial carcinomas (25, Fleming et al, Proc ASCO, 2003). Our data indicate that the proportion of HER-2/neu positive cases also amongst the endometrioid cancers, are sufficient for potential clinical relevance and seems to justify further clinical trials of trastuzumab efficacy in endometrial cancer.

For EGFR, a relationship between the number of receptors and carcinogenesis has been proposed, but the value as an independent prognostic indicator for endometrial carcinoma is not clear (19). The present series has been carefully characterized immunohistochemically with three different EGFR antibodies. The proportion of samples with moderate to strong staining for EGFR was high (33\%), but membranous staining showed no significant associations with clinical phenotype nor survival and is in line with other studies (26). 
High-level amplification of the EGFR gene as estimated by SNP array was also infrequent occurring only in $1.3 \%$ of the samples investigated. Even though this sample showed clear immunohistochemical overexpression of EGFR, the alteration in gene copy numbers estimated by SNP array can not explain the mechanism for overexpression observed in the independent and larger series studied, indicating that other mechanisms may be involved. EGFR staining did not show any prognostic effect in our series, but the relation to response is still an open question.

Our finding that high proliferative activity in tumors is associated with aggressive disease and poor survival is in line with previous studies demonstrating the importance of proliferative activity in endometrial tumors (27). To our knowledge, this is the first study comparing the proliferative activity in tumor cells from curettage and hysterectomy specimens in a population based setting. We found that the estimated proliferative activity in the curettage and hysterectomy specimens was significantly correlated and both were associated with an aggressive phenotype, although a stronger prognostic influence was seen for the estimated proliferation in the hysterectomy specimens. This appears to be in line with one small previous report (28). Estimation of Ki-67 expression in curettage specimens did not add independent prognostic information beyond the traditional prognostic markers in our study, and is insufficient to be used for individualised treatment.

Tumor heterogeneity may explain the differences in estimates for curettage and hysterectomy specimens for both HER-2/neu and Ki-67 expression. Tumor necrosis, which is more likely to be present in an exfoliating part of the exophytic tumor in curettage material, may also affect the estimation of proliferation. Our finding that Ki-67 expression in curettage specimens was significantly correlated to presence of necrosis may support this explanation. Fixation method was the same for both specimen types and fixation time was not significantly different.

In conclusion, high level amplification of HER-2/neu or EGFR is infrequent in endometrial cancer. High expression of HER-2/neu in curettage and hysterectomy specimens from endometrial carcinomas detected immunohistochemically is relatively common and identifies tumors with serous papillary/clear cell histology and high proliferation. Also, high HER/neu expression in tumors from hysterectomy specimens is associated with poor survival. These results motivate clinical trials with trastuzumab based on molecular HER-2/neu status in the tumors. We found in this populationbased endometrial carcinoma study that estimation of proliferative activity (Ki-67 expression) in the hysterectomy specimens is superior to estimation in curettage specimens and add significant independent prognostic information to the established clinico-pathological variables.

\section{Acknowledgements}

Helse Vest Grant $(911069,911351)$ and The Norwegian Cancer Society (D94070/04, D00019/01) supported this study. We thank Gerd Lillian Hallseth, Bendik Nordanger, Britt Edvardsen and Randi Nygaard for excellent technical assistance.

\section{References}

1. Amant F, Moerman P, Neven P, Timmerman D, van Limbergen E and Vergote I: Endometrial cancer. Lancet 366: 491-505, 2005.

2. Jacks T and Weinberg RA: Cell-cycle control and its watchman. Nature 381: 643-644, 1996.

3. Elit L and Hirte H: Current status and future innovations of hormonal agents, chemotherapy and investigational agents in endometrial cancer. Curr Opin Obstet Gynecol 14: 67-73, 2002.

4. Mariani A, Sebo TJ, Katzmann JA, Roche PC, Keeney GL, Lesnick TG and Podratz KC: Endometrial cancer: can nodal status be predicted with curettage? Gynecol Oncol 96: 594-600, 2005 .

5. Gonzalez-Angulo AM, Hortobagyi GN and Esteva FJ: Adjuvant therapy with trastuzumab for HER-2/neu-positive breast cancer. Oncologist 11: 857-867, 2006.

6. Marshall J: Clinical implications of the mechanism of epidermal growth factor receptor inhibitors. Cancer 107: 1207-1218, 2006.

7. Dougall WC, Qian X, Peterson NC, Miller MJ, Samanta A and Greene MI: The neu-oncogene: signal transduction pathways, transformation mechanisms and evolving therapies. Oncogene 9: 2109-2123, 1994

8. Hung MC and Lau YK: Basic science of HER-2/neu: a review. Semin Oncol 26: 51-59, 1999.

9. Santin AD, Bellone S, Gokden M, Palmieri M, Dunn D, Agha J, Roman JJ, Hutchins L, Pecorelli S, O'Brien T, Cannon MJ and Parham GP: Overexpression of HER-2/neu in uterine serous papillary cancer. Clin Cancer Res 8: 1271-1279, 2002.

10. Albitar L, Laidler LL, Abdallah R and Leslie KK: Regulation of signaling phosphoproteins by epidermal growth factor and Iressa (ZD1839) in human endometrial cancer cells that model type I and II tumors. Mol Cancer Ther 4: 1891-1899, 2005.

11. Engelsen IB, Stefansson I, Akslen LA and Salvesen HB: Pathologic expression of p53 or p16 in preoperative curettage specimens identifies high-risk endometrial carcinomas. Am J Obstet Gynecol 195: 979-986, 2006.

12. Salvesen HB, Iversen OE and Akslen LA: Prognostic significance of angiogenesis and $\mathrm{Ki}-67, \mathrm{p} 53$, and p21 expression: a populationbased endometrial carcinoma study. J Clin Oncol 17: 1382-1390, 1999.

13. Stefansson IM, Salvesen HB and Akslen LA: Prognostic impact of alterations in P-cadherin expression and related cell adhesion markers in endometrial cancer. J Clin Oncol 22: 1242-1252, 2004.

14. Lieberfarb ME, Lin M, Lechpammer M, Li C, Tanenbaum DM, Febbo PG, Wright RL, Shim J, Kantoff PW, Loda M, Meyerson M and Sellers WR: Genome-wide loss of heterozygosity analysis from laser capture microdissected prostate cancer using single nucleotide polymorphic allele (SNP) arrays and a novel bioinformatics platform dChipSNP. Cancer Res 63: 4781-4785, 2003.

15. Rhodes A, Jasani B, Anderson E, Dodson AR and Balaton AJ: Evaluation of HER-2/neu immunohistochemical assay sensitivity and scoring on formalin-fixed and paraffin-processed cell lines and breast tumors: a comparative study involving results from laboratories in 21 countries. Am J Clin Pathol 118: 408-417, 2002.

16. Galizia G, Lieto E, Ferraraccio F, De Vita F, Castellano P, Orditura M, Imperatore V, La Mura A, La Manna G, Pinto M, Catalano G, Pignatelli $\mathrm{C}$ and Ciardiello F: Prognostic significance of epidermal growth factor receptor expression in colon cancer patients undergoing curative surgery. Ann Surg Oncol 13: 823-835, 2006.

17. Stefansson IM, Salvesen HB, Immervoll $\mathrm{H}$ and Akslen LA: Prognostic impact of histological grade and vascular invasion compared with tumour cell proliferation in endometrial carcinoma of endometrioid type. Histopathology 44: 472-479, 2004.

18. Lax SF: Molecular genetic pathways in various types of endometrial carcinoma: from a phenotypical to a molecular-based classification. Virchows Arch 444: 213-223, 2004.

19. Khalifa MA, Abdoh AA, Mannel RS, Haraway SD, Walker JL and Min KW: Prognostic utility of epidermal growth factor receptor overexpression in endometrial adenocarcinoma. Cancer 73: 370-376, 1994.

20. Ryan AJ, Susil B, Jobling TW and Oehler MK: Endometrial cancer. Cell Tissue Res 322: 53-61, 2005. 
21. Mariani A, Sebo TJ, Katzmann JA, Riehle DL, Dowdy SC, Keeney GL, Lesnick TG and Podratz KC: HER-2/neu overexpression and hormone dependency in endometrial cancer: analysis of cohort and review of literature. Anticancer Res 25: 2921-2927, 2005.

22. Morrison C, Zanagnolo V, Ramirez N, Cohn DE, Kelbick N, Copeland L, Maxwell GL and Fowler JM: HER-2 is an independent prognostic factor in endometrial cancer: association with outcome in a large cohort of surgically staged patients. J Clin Oncol 24: 2376-2385, 2006.

23. Mariani A, Sebo TJ, Katzmann JA, Keeney GL, Roche PC, Lesnick TG and Podratz KC: Pretreatment assessment of prognostic indicators in endometrial cancer. Am J Obstet Gynecol 182: 1535-1544, 2000.

24. Slomovitz BM, Broaddus RR, Burke TW, Sneige N, Soliman PT, Wu W, Sun CC, Munsell MF, Gershenson DM and Lu KH: Her-2/neu overexpression and amplification in uterine papillary serous carcinoma. J Clin Oncol 22: 3126-3132, 2004.
25. Jewell E, Secord AA, Brotherton T and Berchuck A: Use of trastuzumab in the treatment of metastatic endometrial cancer. Int J Gynecol Cancer 16: 1370-1373, 2006.

26. Reinartz JJ, George E, Lindgren BR and Niehans GA: Expression of $\mathrm{p} 53$, transforming growth factor alpha, epidermal growth factor receptor, and c-erbB-2 in endometrial carcinoma and correlation with survival and known predictors of survival. Hum Pathol 25: 1075-1083, 1994.

27. Salvesen HB, Iversen OE and Akslen LA: Identification of high-risk patients by assessment of nuclear Ki-67 expression in a prospective study of endometrial carcinomas. Clin Cancer Res 4: 2779-2785, 1998

28. Oreskovic S, Babic D, Kalafatic D, Barisic D and BeketicOreskovic L: A significance of immunohistochemical determination of steroid receptors, cell proliferation factor Ki-67 and protein p53 in endometrial carcinoma. Gynecol Oncol 93: 34-40, 2004. 